\title{
OBSERVATIONS OF QUASI-PERIOdic OSCILlations IN CYG X-2
}

\author{
Günther Hasinger \\ Max-Planck-Institut für \\ extraterrestrische Physik \\ 8046 Garching bei München \\ Federal Republic of Germany
}

\begin{abstract}
EXOSAT observations of Cyg X-2 reveal two types of QPO's (quasiperiodic oscillations) which are associated with different spectral behaviour. QPO's in the range 18-55 $\mathrm{Hz}$ with a hard spectrum are found in all observations where the source is on the 'horizontal branch' in an hardness-versus-intensity diagram. On the 'vertical branch', however, QPO's with a soft spectrum are found at a stable frequency of $5.6 \mathrm{~Hz}$. A multitude of QPO frequency-intensity relations, found in different 'horizontal branch' observations, coalesce to an almost unique relation when the QPO frequency is correlated with spectral hardness. A cross-correlation analysis of the rapid variability reveals a time-lag of hard photons by $1.5-3.8 \mathrm{~ms}$; the lag being smaller when the QPO frequency is larger. All results are consistent with a model where QPO's represent the Kepler frequency at the edge of the neutron star magnetosphere. In particular the data do not require the assumption of a beat frequency. The derived surface magnetic field strength is of the order of $10^{10} \mathrm{G}$.
\end{abstract}

\section{Introduction}

Quasi-periodic oscillations on timescales of tens of milliseconds have been discovered in the X-ray flux of bright low-mass X-ray binaries (van der Klis et al., 1985a); up to date the class of QPO sources contains about nine objects. For detailed reviews of the observational material (much of which as yes unpublished) see Lewin and van Paradijs (1986) and van der Klis (1987). Several models have been proposed to explain QPO's (see van der Klis, 1987; Lewin, 1986) but there is no general consensus about the production mechanism. In particular it is unclear, whether QPO's are of magnetospheric origin or not. Today it is obvious that the QPO production mechanism is much more complicated than the early results have suggested. The variety of puzzling phenomena increased with every new observation. It is quite conceivable that more than one production mechanism has to be considered.

In this review an extended body of QPO observations of Cyg X-2 is discussed. The question, what information QPO's contain about the neutron star magnetic field, is adressed. This paper contains some unpublished material of which a more detailed analysis will be presented elsewhere (Hasinger et al., 1986b). 


\section{Two spectral modes}

\subsection{Hardness -versus-intensity diagrams}

Six observations of Cyg X-2 have been carried out with the Medium Energy Detectors (Turner et al., 1981) aboard the European Space Agency's X-ray Observatory EXOSAT, yielding a total net observing time of $3 \cdot 10^{5} \mathrm{~s}$. Figure 1 shows hardnessversus-intensity diagrams for all observations. The data points are samples of $200 \mathrm{~s}$, the different symbols refer to different observations. The abscissa gives total (1-17 $\mathrm{keV}$ ) source count rates, corrected for deadtime and background, and normalized to 8 observing detectors $\left(\sim 1500 \mathrm{~cm}^{2}\right)$.

The ordinate gives the hardness ratios between two energy channels: $(6-17 / 3-6$ $\mathrm{keV}$ ) for figure 1a and (3-6/1-3 keV) for figure 1b. Although the source was found in several distinct tracks in this diagram, there are only two characteristic modes of spectral behaviour, as was first noted by Branduardi et al. (1979). On the 'horizontal branch' (Shibazaki and Mitsuda, 1984) in figure 1a the 'hard' hardness ratio $(6-17 / 3-6 \mathrm{keV})$ changes only very little with increasing source intensity. Figure $1 \mathrm{~b}$, where the 'soft' hardness ratio (3-6/1-3 keV) is plotted against intensity, demonstrates that the form of the tracks in this diagram is dependent on the choice of energy bands. The tracks of the 'horizontal' branch observations are now actually inclined, i.e. hardness increases with source intensity.

On the 'vertical' branch (which is usually called the 'normal' branch), on the other hand, there is a steep gradient in hardness ratio, which varies dramatically during small intensity changes. This is due to the fact that intensity variations in the high and low bands are anticorrelated (see also Hasinger et al., 1985a), so that decreases at low energies are cancelled by increases at high energies and vice versa. Similar 'vertical' branch behaviour has been reported for Cyg X-2 by Vrtilek et al. (1986).

Transitions between these two modes have been observed several times; they happen on a timescale of $\sim 1000 \mathrm{~s}$.

The differences between figure 1 and 'classical' hardness- intensity diagrams (Branduardi et al., 1979; Shibazaki and Mitsuda, 1983; van der Klis, 1987) are probably only due to the difference in energy bandpass for different measurements: the 1-2 keV range is not accessible for most detectors, nor for heavily absorbed sources.

\subsection{Spectral decomposition}

Analysis of the source spectra in terms of a two-component model (Comptonized thermal and blackbody) indicates, that on the 'horizontal' branch the blackbody component is responsible for most of the intensity variations, while the thermal component stays nearly constant in intensity and temperature (Hasinger et al., 1986a). Consequently the soft hardness ratio (fig. 1b) is a good measure of the 


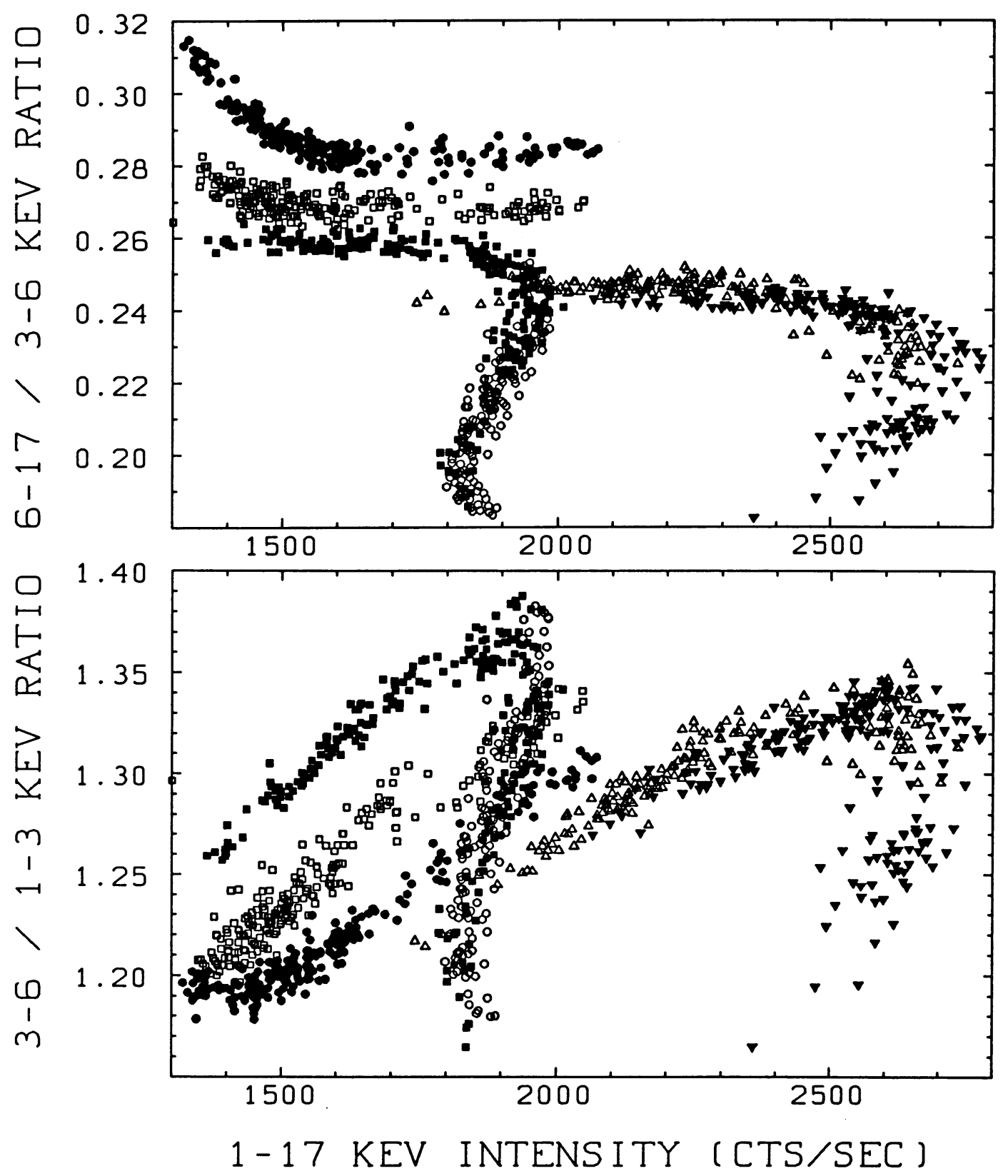

Figure 1: Hardness-versus-intensity diagrams for Cyg X-2. Top panel (fig. 1a): 'hard' hardness ratio; bottom panel (fig. 1b): 'soft' hardness ratio.

blackbody contribution in the 'horizontal' branch.

On the 'vertical' branch, however, preliminary analysis indicates, that the blackbody component stays roughly constant around the highest level observed in the 'horizontal' branch (Hasinger et al., 1986b). The large hardness changes are here 
due to variations in the slope of the 'thermal' component, which tilts around a pivotpoint at $\sim 3 \mathrm{keV}$. This results roughly in a net conservation of the number of photons in the observed band (1-17 keV), a behaviour very suggestive for Compton scattering through a cloud of variable optical depth.

\section{QPO analysis}

Every observation in figure 1 has been subdivided into several groups of data points at similar intensity and hardness. For these observation subsets the spectral and timing properties of the source were assumed to be constant, so that power spectra could be averaged to perform a 'standard' QPO analysis (van der Klis et al., 1985a; Hasinger et al., 1986a).

\section{1 'Horizontal' branch}

The 'classical', intensity-dependent QPO's, that have been discovered from GX 5-1 (van der Klis et al., 1985) and subsequently from Cyg X-2 (Hasinger et al., 1986a), are observed exclusively during 'horizontal' branch behaviour (see also van der Klis et al., 1985b). Figure $2 a$ shows the resulting power spectra for a typical 'horizontal' branch observation. The average source intensity increases from top to bottom. QPO's and LFN (low-frequency noise) are clearly visible in each panel. The QPO frequency increases from 18 to $45 \mathrm{~Hz}$ with increasing source intensity. At low intensities there is a clear indication of additional QPO power at around twice the frequency of the fundamental peak, characteristic of higher harmonics. However, in the second panel (where the addidional peak is most prominent) a fit with two Lorentzian peak profiles yields a frequency ratio of $1.85 \pm 0.03$, smaller than that expected for a second harmonic.

Utilizing an energy-resolved high-time-resolution mode of the EXOSAT onboard computer specifically designed for QPO research (HER7), one can determine the spectral properties of the oscillations. Averaging all data from figure 2a, one finds that in the 'horizontal' branch QPO (and LFN) are much harder than the mean source spectrum: the rms. relative equivalent sinusoidal modulation increases strongly with energy from $\sim 4$ percent at $1 \mathrm{keV}$ to $\sim 10$ percent at $10 \mathrm{keV}$.

\section{2 'Vertical' branch}

In a preliminary analysis of GX 5-1 no QPO's were found on the 'vertical' branch (van der Klis et al., 1985b). However, for Cyg X-2 QPO's have been found in the 'vertical' branch, though with properties completely different from those in the 'horizontal' branch. Figure $2 \mathrm{~b}$ shows power spectra averaged for a 'vertical' branch observation. The mean spectral hardness decreases from top to bottom. QPO's at a frequency of $5.6 \mathrm{~Hz}$ are observed, but only in the panels 2 and 3, i.e. exclusively at intermediate hardness ratios. Similar QPO's have been observed in two other EXOSAT 'vertical' branch observations (Hasinger et al., 1986b), and by HEAO-1 (Norris and Wood, 1985). In all cases the observed frequency is consistent with 


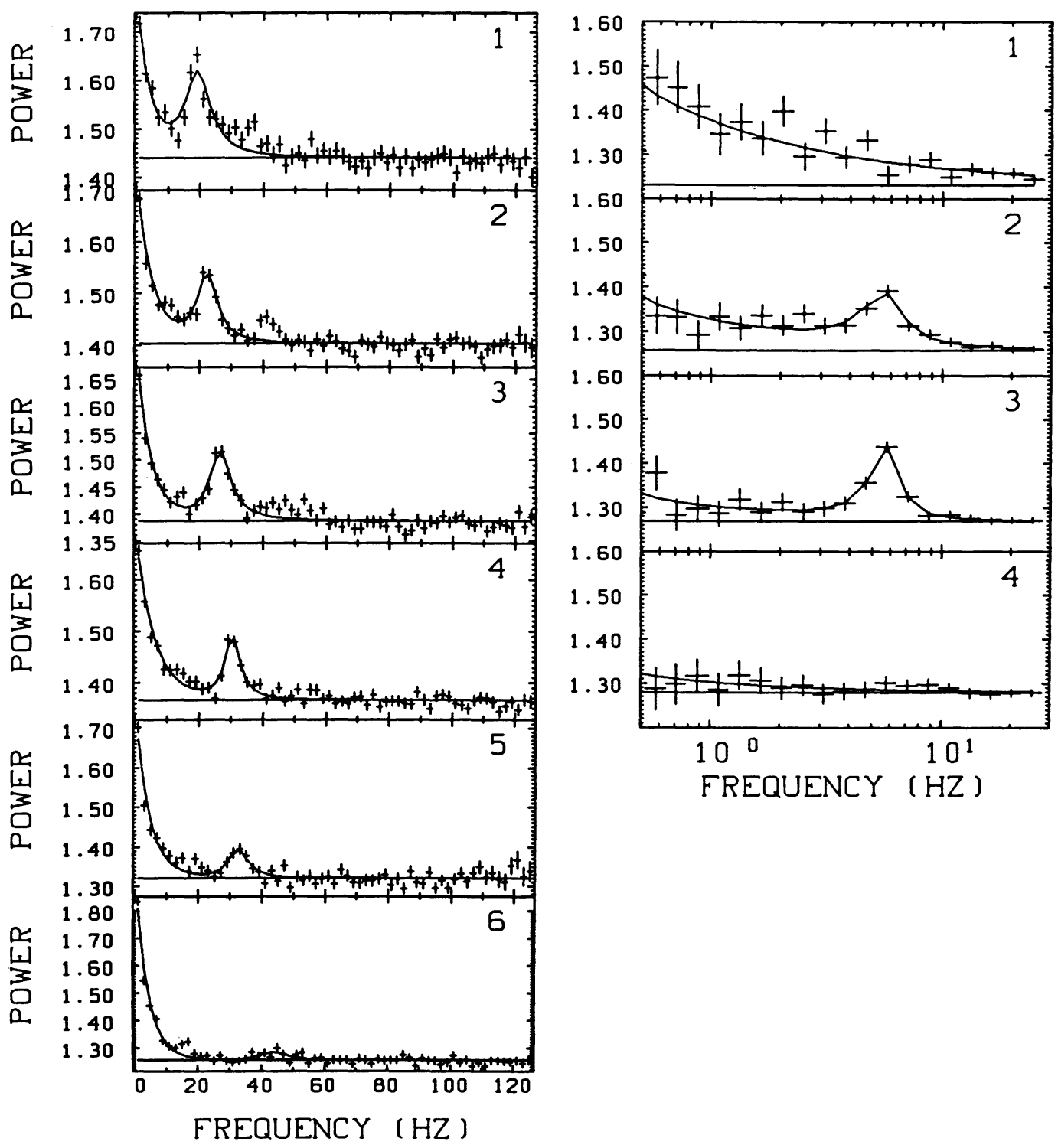

Figure 2: Power spectra of Cyg X-2. 2a (left): A 'horizontal' branch observation; the source intensity increases from $1354 \mathrm{cts} / \mathrm{sec}$ in panel 1 to $1990 \mathrm{cts} / \mathrm{sec}$ in panel 6. 2b (right): A 'vertical' branch observation; the 'soft' hardness ratio decreases from 1.36 in panel 1 to 1.20 in panel 4.

a value of $5.6 \mathrm{~Hz}$. The remarkable stability of this frequency over timescales of months to years is contrasted by a highly time-variable QPO occurence, which is a consequence of their restriction to a certain band in spectral hardness.

Another characteristic difference compared to the 'horizontal' branch behaviour is the weakness of the low-frequency noise during times when QPO are observed. The LFN decreases steadily with decreasing hardness ratio. 
The most distinct difference, however, is the QPO spectrum: on the 'vertical' branch the QPO's are soft: the rms. relative amplitude is $\sim 4$ percent at $1 \mathrm{keV}$ and drops below 2.3 percent above $4.5 \mathrm{keV}$.

Recently, an indication for $\sim 5 \mathrm{~Hz}$ QPO's has also been found in the 'vertical' branch of GX 5-1 (van der Klis, 1987). Taking into account all the differences between 'vertical' and 'horizontal' branch QPO's, it seems very likely that they are produced by different mechanisms or in different geometries. One has therefore to be careful to compare all the properties of QPO's in different sources.

\section{Which quantity determines the QPO frequency ?}

The further discussion will be limited to the 'classical', hard QPO's in the 'horizontal' branch which, due to their strong intensity-dependence enable a comparison to model predictions. The sources GX 5-1, Cyg X-2 and, as will be argued in the following, Sco X-1 belong into this class.

\subsection{The source spectrum}

One of the most striking facts of the early QPO discoveries was the strong intensity dependence of the QPO frequency. In GX 5-1, Cyg X-2 and at times Sco $\mathrm{X}-1$ the frequency $f$ was a steep function of intensity $I$ with a logarithmic slope $\alpha=d \log (f) / \operatorname{dlog}(I)=1.7-3$ (van der Klis et al., 1985a,b; Hasinger et al., 1986). The simplicity and uniqueness of this behaviour led to the tentative interpretation of the QPO's as the result of a chaotic process at the boundary of the neutron star magnetosphere (Alpar and Shaham, 1985; van der Klis et al. 1985a).

With the subsequent analysis of more observations, it became clear that a variety of frequency-intensity relationships is possible. Sco X-1 displays a continuous transition from a strongly positive to an erratic and then a slightly negative correlation between frequency and intensity as the source marches through a bimodal hardness-intensity diagram (Priedhorsky et al., 1986; van der Klis, 1987). Cyg $\mathrm{X}-2$, on the other hand, falls on different tracks on the intensity-frequency plane for every other observation (figure 3a), displaying a similar ambiguity as in the hardness-intensity plane (see figure 1). In both sources, Cyg X-2 and Sco X-1 (see also Priedhorsky, 1986) it seems that the total X-ray intensity is an ill-behaving parameter and that a much simpler description of the QPO properties can be found via the source spectrum.

In figure $3 b$ the QPO frequency is correlated to the 'soft' hardness ratio (3-6/1$3 \mathrm{keV}$ ) for all horizontal branch observations of Cyg X-2. The individual tracks for different observation coalesce to one, nearly unique relationship in this representation. (This is strictly true for frequencies below $40 \mathrm{~Hz}$, the observation at the highest intensities is slightly offset, possibly because of a hardness variation in the soft component). One therefore has to conclude, that the shape of the X-ray spectrum is a much better indicator of the QPO frequency than the total X-ray intensity. In a two-component spectral fit the soft hardness ratio is a measure of the 


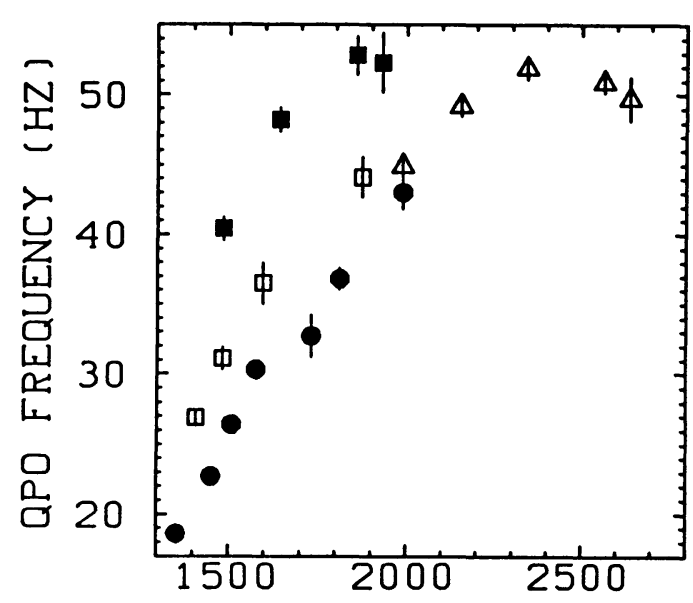

INTENS ITY (CTS/SEC)

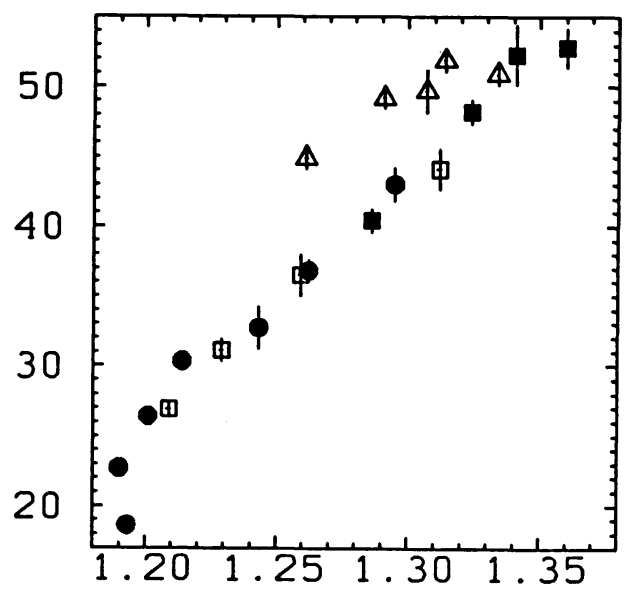

HARDNESS $(3-6 / 1-3 \mathrm{KEV})$

Figure 3: Correlation of the QPO frequenies from 'horizontal' branch observations of Cyg X-2. Left panel (fig. 3a): frequency versus intensity; right panel (fig. 3a): frequency versus 'soft' hardness ratio.

blackbody contribution (see above), and preliminary spectral fits show indeed, that the QPO frequency is better related with the blackbody flux than with the total $\mathrm{X}$-ray flux (Hasinger, 1985). Due to the relatively large variations of the blackbody component the QPO frequency is a rather flat function of the blackbody flux $F_{b b}$ : the logarithmic derivative $\alpha_{b b}=\operatorname{dlog}(f) / \operatorname{dlog}\left(F_{b b}\right)$ is only $\sim 0.5$ for Cyg X-2 (Hasinger et al., 1986a). This is consistent with the value $\alpha=3 / 7$ expected for the Kepler frequency at the boundary of the neutron star magnetosphere (Bath, 1973).

Also for Sco X-1 the complicated bimodal spectral and QPO behaviour reduces to a rather simple dependence of QPO frequency on blackbody flux (van der Klis, 1986). Together with the fact that all QPO's from Sco X-1 have a hard spectrum (Priedhorsky et al., 1986) this indicates that there is no principal difference to the QPO's in GX 5-1 and Cyg X-2 on one hand and Sco X-1 on the other hand.

The decomposition of low-mass X-ray binary spectra into two simple components is the subject of some controversy; the true source spectra are certainly more complicated. The limited bandwidth of X-ray observations, the rather coarse spectral resolution, the interstellar absorption and other systematic effects lead to ambiguities in the parameter estimation. In addition the decomposition is not unique, and different schools of thought prefer different representations. In particular there is no consensus regarding the 'thermal' or 'soft' component, which is modelled as a 'multitemperature blackbody' by the TENMA team (Mitsuda et al., 1984) and as 'unsaturated Comptonization' by EXOSAT observers (White et al., 1986). Consequently the derived blackbody temperatures and fluxes are very different in the two representations.

The strong correlation of an independent parameter, namely the QPO frequency, to the blackbody flux is supporting the 'unsaturated Comptonization' model, and may indicate that the blackbody flux is a real physical quantity and not just the figment of a mathematical parametrization. 


\subsection{The size of the X-ray emitting region}

The ambiguities in total intensity and source spectra outlined above, make it desirable to find an independent physical quantity to describe the QPO frequency behaviour. Until now QPO research was mainly based on power spectral analysis, which maintains the frequency information but throws away all phase information involved in the quasi-periodic process. With the use of the energy-resolved high-time-resolution mode of the EXOSAT on-board computer (HER7), a crosscorrelation analysis between the light curves in different energy bands can be performed.

The model of a Comptonized spectrum used for the spectral decomposition (see above) makes an important prediction: soft photons are upscattered in a cloud of hot electrons, thus the rapid fluctuations at high energies should lag behind those at low energies. The time-lag is an indirect measure of the size of the scattering cloud, and is expected to be on the order of several milliseconds (Shapiro et al., 1976; Priedhorsky et al., 1979; Page, 1985).

For Cyg X-2 subsamples of 'horizontal' branch observations at roughly the same QPO frequency have been selected (typically 12-16 s). The light curve in the highenergy band (4.5-17 keV) was cross-correlated with the one in the low-energy band (1-4.5 keV). The cross-correlation function (CCF) was derived by averaging the results from many of these subsets. Figure 4 displays a CCF, obtained by averaging $\sim 38400 \mathrm{~s}$ of data with a time resolution of $3.91 \mathrm{~ms}$. Error bars are estimated from the variance of 2401 subsamples. The CCF is asymmetric, indicating that the average properties of the rapid fluctuations are different in the two energy bands. In addition the CCF has sinusoidal ondulations which are due to the quasi-periodic modulation. The most important finding is, however, a time lag of hard versus soft photons. This is clearly visible for the data points at $\pm 3.91 \mathrm{~ms}$, which should have roughly the same height for a zero lag.

The solid line represents a fit to the data of the form:

$$
\begin{array}{ll}
C C F=A e^{-\frac{t-\Delta}{\tau_{1}}}(1+B \cos (\omega(t-\Delta)))+C & \text { for } t>\Delta \text { and } \\
C C F=A e^{-\frac{\Delta-t}{\tau_{2}}}(1+B \cos (\omega(\Delta-t)))+C & \text { for } t<\Delta,
\end{array}
$$

i.e. a delayed, asymmetrically decaying exponential function modulated by a delayed, exponentially decaying cosine. $A, B$ and $C$ are normalization constants, $\tau_{1}$ and $\tau_{2}$ are the exponential decay times for positive and negative lags, respectively, $\omega$ is the QPO frequency and $\Delta$ is the time delay. The significance of the parameter $\Delta$ has been estimated with an F-test (Bevington, 1969): the same fit as above was applied to the data, but $\Delta$ was forced to zero. Comparing the reduction in $\chi^{2}$ the significance of the additional parameter was estimated.

Since a large amount of data had to be averaged for every single CCF and since the HER7 mode was not available for earlier EXOSAT observations, only three independent CCFs could be derived for Cyg X-2. Table 1 summarizes the results of these 


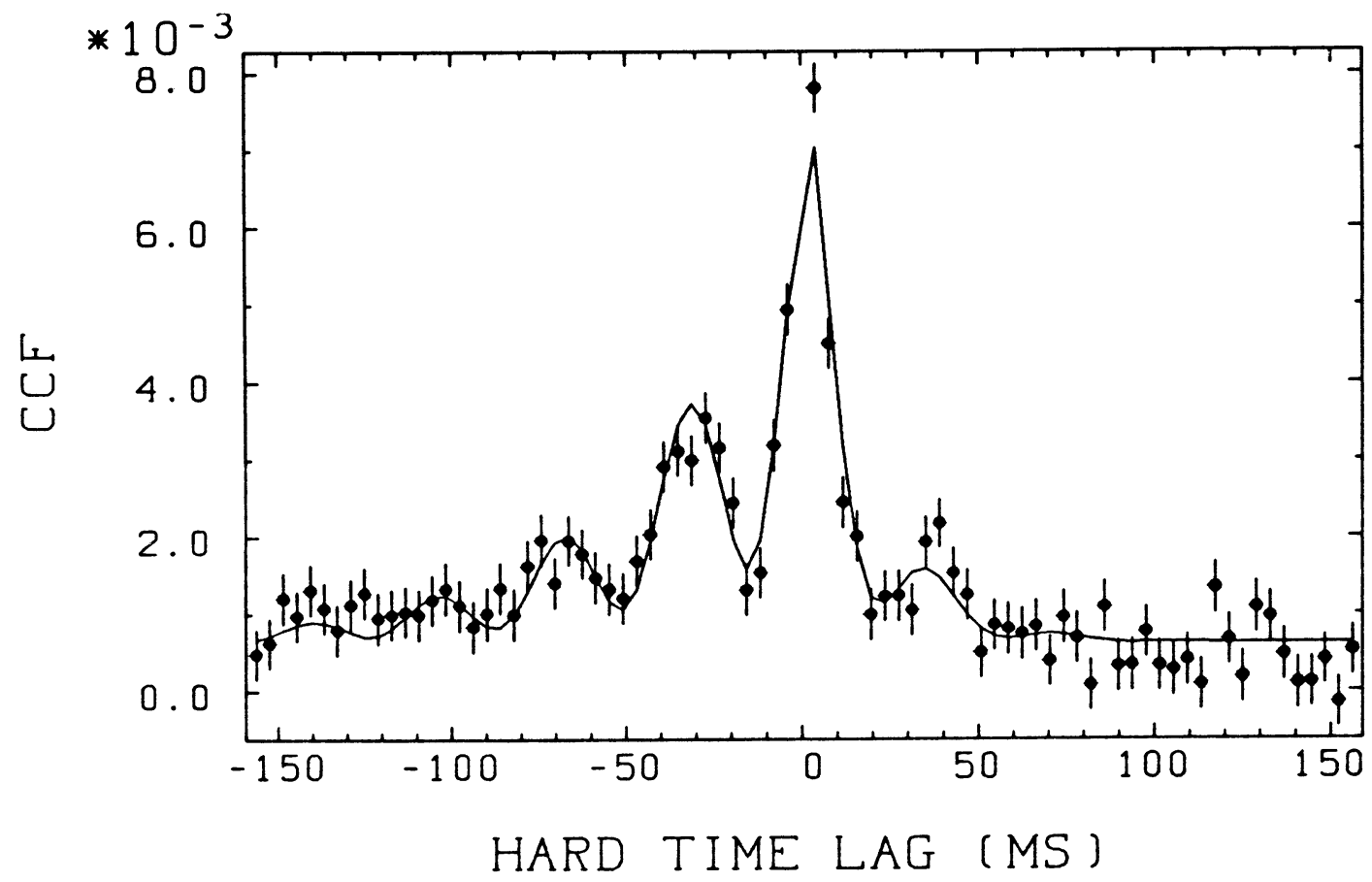

Figure 4: Sample cross-correlation function for Cyg X-2. Data points are seperated by $3.91 \mathrm{ms.}$ The error bars are standard deviations derived by the variance of the 2401 samples averaged.

time-lag measurements at different QPO frequencies. Column 1 gives the mean QPO frequency and column 2 the measured time lag. Errors are standard deviations for one parameter of interest (Avni, 1976). Column 3 gives the time-resolution for the relevant measurement and columns 4, 5 and 6 give the F-value for the time lag, the probability of this value occurring by chance and the derived significance of the lag value.

\begin{tabular}{|c|c|c|c|c|c|}
\hline $\begin{array}{c}\text { Frequency } \\
(\mathrm{Hz})\end{array}$ & $\begin{array}{c}\text { Time lag } \\
(\mathrm{ms})\end{array}$ & $\begin{array}{c}\text { Bin } \\
(\mathrm{ms})\end{array}$ & F-value & Prob. & $\begin{array}{c}\text { Sign. } \\
\sigma\end{array}$ \\
\hline $20.7 \pm 0.3$ & $3.8 \pm 0.3$ & 3.91 & 62.2 & $4.8 \cdot 10^{-9}$ & $>6.6$ \\
$28.4 \pm 0.3$ & $2.7 \pm 0.3$ & 3.91 & 34.3 & $1.1 \cdot 10^{-7}$ & 5.2 \\
$50.4 \pm 1.9$ & $1.4 \pm 0.3$ & 2.92 & 13.5 & $8.6 \cdot 10^{-4}$ & 3.3 \\
\hline
\end{tabular}

Table 1: Results of the cross-correlation analysis for Cyg X-2.

\section{Consequences for QPO models}

The measured time delay is in the right sense, i.e. hard fluctuations lag, in agreement with the Comptonization model. The detailed CCF's provide a variety of onstraints on the quasi-periodic process. The asymmetry of the profiles gives di- 
rect evidence that the average lifetime of the fluctuations is different at high and low energies. The fact that the sidelobes on the CCF also seem to be delayed indicates that the QPO's are formed inside the Compton scattering cloud. The time delay $\Delta$ gives a measure of the size $r_{s}$ of the scatterer: $\Delta=\frac{r_{c} \cdot \tau_{c}}{c} ; \tau_{c}$ being the Compton optical depth and $c$ the velocity of light. With an optical depth of $\tau_{c}=5$ (which is consistent with the Comptonized spectral fits), we find $r_{s} \approx 170 \mathrm{~km}$ for a time lag of $3 \mathrm{~ms}$. At this radius the Kepler frequency of material orbiting the neutron star is $\sim 20 \mathrm{~Hz}$, i.e. consistent with the observed QPO frequency.

The most important finding is, however, that the time-lag gets smaller for larger QPO frequency, as displayed in figure 5. This is the first independent indication that a change in QPO frequency is really associated with a considerable change in the system size, placing severe constraints on QPO models. The measured data points are consistent with a power law of a slope $-2 / 3$ (solid line in figure 5 ), which is the expected dependence of the time delay on the QPO frequency under the assumption that the QPO frequency is identical to the Kepler frequency at $r_{s}$, and that $\tau_{c}$ stays roughly constant. The slope implied by the data is even somewhat steeper.

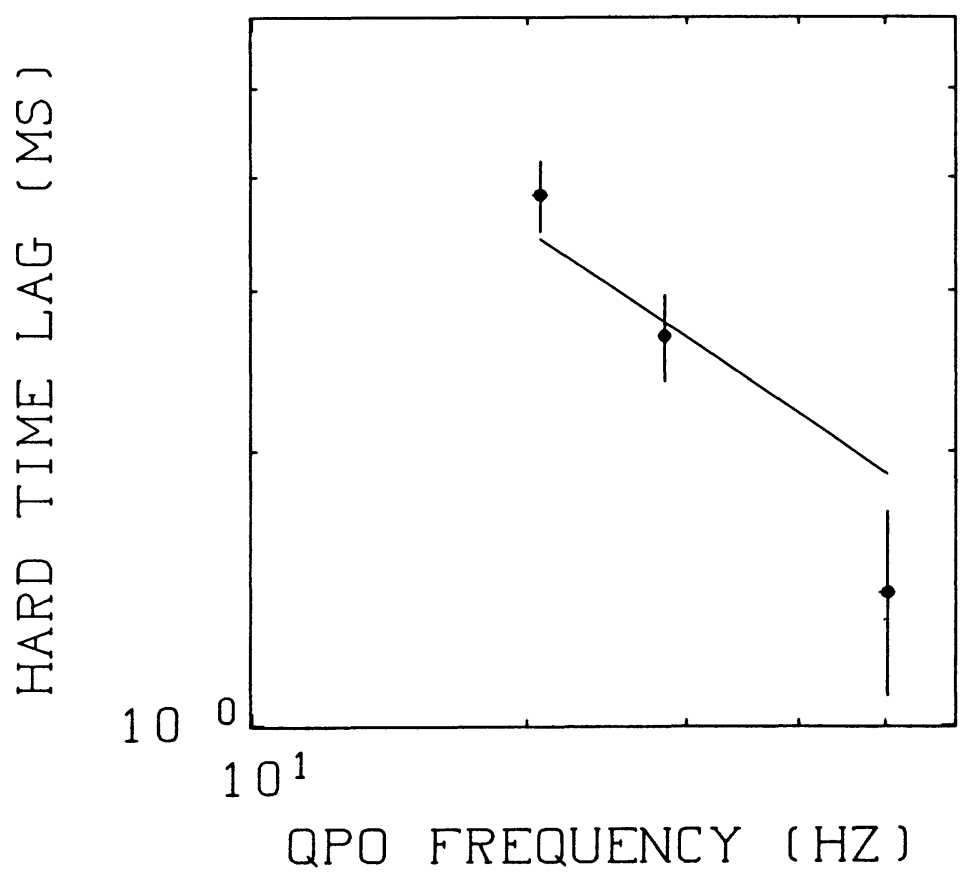

Figure 5: Time delay as a function of QPO frequency for Cyg X-2. The solid line is the dependence expected for the Kepler frequency.

Although the exact mechanism that produces QPO's is far from understood, the new observational results of Cyg X-2 strongly favour the view that in the 'horizontal branch' we are dealing with the Kepler frequency at the magnetospheric boundary. The dependence of QPO frequency on the blackbody luminosity and independently the relation of the time delay on QPO frequency are both perfectly 
consistent with this conception. The assumption of addidional parameters, as e.g. in the beat-frequency model (Alpar and Shaham, 1985; Lamb et al., 1985), seem unnecessary. Trying to estimate the surface magnetic field of the neutron star in the framework of the Bath model (van der Klis et al., 1985a; Hasinger et al., $1986 \mathrm{a})$ there is a remaining ambiguity as to which value for the mass accretion rate $\dot{M}$ should be taken. The ill-behaved total luminosity is certainly not a good measure for $\dot{M}$. The blackbody luminosity, on the other hand, seems to be proportional to the mass accretion rate, but the absolute value of $\dot{M}$ remains unknown. Thus only a rough estimate of the magnetic field $\left(B \approx 10^{10} G\right)$ can be derived.

\section{Acknowledgements}

I thank my collaborators in this project: Andreas Langmeier, Drs. Mirek Sztajno, Wolfgang Pietsch, Manfred Gottwald and Bill Priedhorsky. I acknowledge clarifying discussions with Drs. Jan van Paradijs and Frank Verbunt, and I thank the organizers of the 125th IAU symposium in Nanjing, China for an enchanting meeting.

\section{References}

Avni, Y. 1976, Astrophys. J. $210,642$.

Alpar, M.A. and Shaham, J. 1985, Nature 316, 239.

Bath, G.T. 1973, Nature Phys. Sci 246, 84.

Bevington, P.R. 1969, in: Data Reduction and Error Analysis for the Physical Sciences (New York: McGraw Hill).

Branduardi, G., Kylafis, N.D., Lamb, D.Q. and Mason, K.O. 1980, Astrophys. J. (Letters) 235, L153.

Hasinger, G. 1985, talk at the 'Discussion Group on EXOSAT Results', Cambridge, England, November 1985.

Hasinger, G., Langmeier, A., Pietsch, W. and Sztajno, M. 1985a Space Sci. Rev. 40, 233.

Hasinger, G., Langmeier, A., Sztajno, M., Pietsch, W. and Gottwald, M. 1985b, IAU Circ. No. 4153.

Hasinger, G., Langmeier, A., Sztajno, M., Trümper, J., Lewin, W.H.G. and White, N.E. 1986a, Nature 319, 469.

Hasinger, G., Langmeier, A., Sztajno, M., Pietsch, W., Gottwald, M. and Priedhorsky, W. 1986b (in preparation)

Lamb, F.K., Shibazaki, N., Shaham, J. and Alpar, M.A. 1985, Nature 317, 681.

Lewin, W.H.G. 1987, this volume.

Lewin, W.H.G. and van Paradijs, J. 1986, Comments on Astrophysics (in press). 
Mitsuda K. et al. 1984, Publ. astr. Soc. Jap. 36, 741.

Norris, J.P. and Wood, K.S. 1985, IAU Circ. No. 4087.

Page, C.G. 1985, Space Sci. Rev. 40, 387.

Priedhorsky, W., Garmire, G.P., Rothschild, R., Boldt, E., Serlemitsos, P. and Holt, S. 1979, Astrophys. J. 233, 350.

Priedhorsky, W. 1986, Astrophys. J. (Letters) (in press).

Priedhorsky, W., Hasinger, G., Lewin, W.H.G., Middleditch, J., Parmar, A. Stella, L. and White, N.E. 1986, Astrophys. J. (Letters) (in press).

Shapiro, S.L., Lightman, A.P. and Eardley, D.M. 1976, Astrophys. J. 204, 187.

Shibazaki, N. and Mitsuda, K. 1984, in: High Energy Transients in Astrophysics, ed. S.E.Woosley, p.49, American Institute of Physics, New York.

Turner, M.J.L., Smith, A. and Zimmermann, H.U. 1981, Space Sci. Rev. 30, 513. van der Klis, M., Jansen, F., van Paradijs, J., Lewin, W.H.G., Trümper, J., van den Heuvel, E.P.J. and Sztajno M. 1985a, Nature 316, 225.

van der Klis, M., Jansen, F., van Paradijs, J., Lewin, W.H.G., Trümper, J. and Sztajno, M. 1985b, IAU Circ. No. 4140.

van der Klis, M. 1987 this volume.

van der Klis, M., Jansen, F., White, N.E. and Stella, L. 1985, IAU Circ. No. 4068.

Vrtilek, S.D., Kahn, S.M., Grindlay, J.E., Helfand, D.J. and Seward, F.D. 1985, Astrophys. J. (in press).

White, N.E., Peacock, A., Hasinger, G., Mason, K.O., Manzo, G., Taylor, B.G., Branduardi-Raymont, G. 1986, Mon. Not. R. astr. Soc. 218, 487.

\section{DISCUSSION}

D. Eichler: What is the electron temperature of your Comptonizing bal1?

G. Hasinger: 3 to $4 \mathrm{KeV}$.

M. van der Rlis: Did you also observe a delay in the $5 \mathrm{~ms}$ QPO?

G. Hasinger: No, the statistics are not yet good enough. The intrinsic time structure in the vertical branch is too long ( 20 ms) to find an expected $10 \mathrm{~ms}$ delay.

W. Sieber: How does the LFN fit into your shot-noise model?

G. Hasinger: Since I get a good fit to the Autocorrelation Function, and the ACF contains the same information as the Power Spectrum, the LFN in the power spectrum is reproduced by the simulations.

N. White: As you point out, the hardness ratio versus intensity plot you get is very sensitive to how you choose your energy bands. This makes comparison between different sources very difficult and perhaps misleading. I would strongly urge that these plots be used $2 n 1 y$ as guides and that more emphasis be given to using the spectral deconvolution when comparing different sources. 
G. Hasinger: I admit that it is hard to compare the Hardness ratio vs. Intensity diagram for different sources 1) because of selection of energy bands and effective bandwidth of the detectors used and 2) because of the effect of interstellar absorption. But spectral fits are model dependent, and as long as we do not agree on using the same spectral model, the results are even less comparable.

N. White: With regard to the screening of millisecond pulsations, John Middleditch pointed out at the Taos meeting last summer (1985) that millisecond pulsations will still survive at an amplitude of a few percent even if the pulsar is in an optically thick cloud.

G. Hasinger: The simulation of J. Middleditch and B. Priedhorsky, to my knowledge, used an infinitely thin slab of finite Compton optical depth, thus taking into account the degradation in intrinsic beaming, but not taking into account the light travel time effects. 